\title{
Unit Commitment of Generator Sets During Dynamic Positioning Operation Based on Consequence Simulation *
}

\author{
Torstein I. B $\emptyset^{*}$ Tor Arne Johansen* Eirik Mathiesen** \\ * Center for Autonomous Marine Operations and Systems, Department \\ of Engineering Cybernetics, Norwegian University of Science and \\ Technology, 7491 Trondheim, Norway (e-mail: torstein.bo@itk.ntnu.no, \\ tor.arne.johansen@itk.ntnu.no). \\ ** Kongsberg Maritime AS, 3616 Kongsberg, Norway \\ (e-mail: eirik.mathiesen@kongsberg.com)
}

\begin{abstract}
For vessels with dynamic positioning system, diesel-electric propulsion is often used. At all time the vessel should be able to withstand any single point failure without loss of position. This paper studies the use of simulation of worst-case failure to decide which configurations of the power plant are sufficient for the current operation. The loss of position due to reduced power to the thrusters is simulated and compared with the safety requirements. This method gives both a practical approach to implement the safety requirement and information which can be used as a decision support system or for automatic start and stop of generator sets. The operational cost is also optimized by using the results from the simulations.
\end{abstract}

\section{INTRODUCTION}

During the last decades diesel-electric propulsion has become industry standard for some types of vessels such as drilling rigs and offshore service vessels. With diesel electric propulsion, the generator sets (diesel engine connected with a generator) produce electrical power and this power is then used by the electrical propulsion units to produce thrust. The power is also used by other consumers, such as drilling drives and hotel loads. This system is highly flexible since generator sets can be started and stopped as the need for power changes, hence the diesel engines can run more efficiently.

Dynamic positioning system (DP) is used to keep a vessel at a fixed position and/or heading. For vessels with dynamic positioning systems of equipment class 2 or 3 , there is a requirement that any single point failure should not propagate to a loss of position (International Maritime Organization, 1994). This is mainly handled by using redundant systems, which make sure that if one subsystem fails, another subsystem is ready to take over the function or the remaining fault-free subsystems provide sufficient capacity.

The number of diesel engines is important for the redundancy of diesel electric systems. The plant must be ready for any failures, such as sudden disconnection of a generator or short circuit in a switchboard. However, the highest efficiency of a typical diesel engine is achieved when it delivers $80 \%$ of its rated power. Other problems do also arise when the utilization is low, such as soothing, extra maintenance due to extra running hours, and

* The authors of this paper are a part of Design to verification of control systems for safe and energy efficient vessels with hybrid power plants (D2V) and are funded by the Research Council of Norway, Kongsberg Maritime, and Det Norske Veritas (DNV). some $\mathrm{NO}_{\mathrm{X}}$ reduction systems are inefficient at low temperature (Realfsen, 2009). It is therefore desired to use as few engines as possible to avoid low-load problems, but enough to still be safe.

The unit commitment problem is to commit the correct units, such that the operational cost is as small as possible given the prediction of the load. The load demand of onshore power generation is very predictable and the number of generators is large (Radan, 2008). A method for selecting the correct units must therefore be used, as testing all combinations is too expensive to calculate. However, for marine power plants are the number of units small, but the loads can be unpredictable. A disconnection of a generator will also give much larger impact, since the number of units is small.

The focus for unit commitment of onshore power generation has mainly been to establish an efficient method to find the optimal solution, given the large set of possible solutions. A normal procedure is to use priority tables, this is a table listing the sequence in which the generators should be started (Lowery, 1966). This list can be made by simply ordering the generators by efficiency. Other approaches using optimization theory are also suggested, by among Lowery (1966); Dillon et al. (1978); Juste et al. (1999); Senjyu and Shimabukuro (2003).

Diesel-wind power stations on island have some of the same challenges as marine power plants. The wind speed is random, which gives a varying power production from the wind turbines and this must be compensated by the diesel generators. Contaxis and Kabouris (1991) present a method for unit commitment where ARMA models are used to predict the power generation from wind turbines. In Dokopoulos and Saramourtsis (1996), Monte Carlo simulations are used to make sure that the plant is able to 
withstand faults of generators; however, the prediction of load and wind is assumed to be perfect.

On marine vessels, generators are committed manually or automatically by load dependent start and stop tables (Adnanes, 2003). This is a priority table, with time and power limits for when a new generator should be started or stopped. This means that a new generator will be started if the power demand goes above a certain limit for some time. Conversely, a generator is stopped if power demand drops below another limit for a certain timespan. Radan et al. (2005) optimizes the load dependent start tables with respect to fuel consumption and constrained by the safety requirement that a disconnection of a generator should not lead to blackout. Radan et al. (2006) suggest algorithms to optimize the load dependent start and stop tables, based on the probability for each operational mode of the vessel.

A challenge is that the safety requirement changes with different operational modes. For example, for a diving vessel safety is much more important during diving operation than during transit. Some vessels have different start/stop tables for each mode and also a minimum number of generator sets for some modes, to handle the changing requirements.

Some operators choose to increase the safety margin by committing additional generator sets. The reason may be that the operator does not have enough information so that they could trust the suggestion given by the auto start/stop table.

Consequence analysis is already required in DP operation with high safety requirements (Det Norske Veritas, 2011). This analysis calculates if the mean thruster force and moment can be achieved after any single failure. This is a static analysis and among the typical fault cases are loss of one switchboard and loss of one engine room. The analysis will also detect a need of starting additional generator sets.

\subsection{Main Contribution}

This article proposes a new method for unit commitment of generator sets for DP vessels. The method uses simulations to check which configurations satisfy the safety requirements. The operational cost of each configuration is then evaluated and the configuration with the lowest cost is selected. The method is a practical approach which is highly configurable with respect to different plants, safety requirements, and operational modes. The method can also be used as a decision support system by presenting the results from the simulations to the operator.

\section{CONSEQUENCE BASED UNIT COMMITMENT}

The idea of the controller is that simulations of failure scenarios are used to check which configurations satisfy the operational requirements. This is done by simulating the response of the vessel for each configuration in the event of the worst-case failure. Then the operational cost is evaluated for each configuration. At last, the configuration which is safe and has the lowest operational cost is suggested as the best configuration. Smarter strategies for selecting test configurations can also be used, if the number

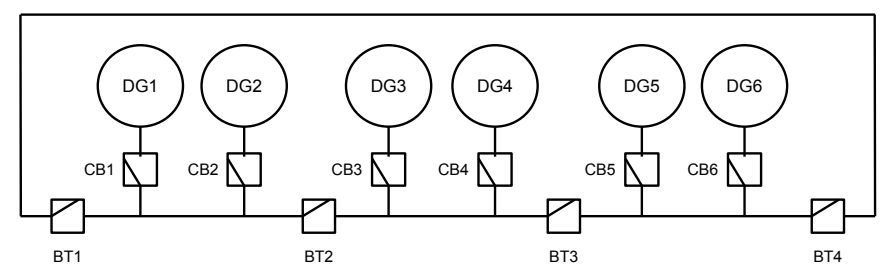

Fig. 1. Single line diagram of case power plant.

of configurations is large and it is too expensive to test all configurations.

The load can be unpredictable; therefore a conservative load may be used in the simulation to make sure that the fault cases include the worst load-case. It is also possible to simulate multiple load cases, such that a broader span of uncertainties can be included without making the solution too conservative. Note that the optimization of operational cost should be done using the most probable prediction, and not the worst-case failure prediction.

\section{CASE STUDY: DP IN STATIC CONDITIONS}

The D2V Case A is used in the case study. This is a drilling ship with six 9.1 MW generator sets. The vessel is simulated in DP with constant ocean current and wind forces. Only the position in surge is simulated; however, the controller can be extended to include any degree of freedom. The power plant is shown in Figure 1, it consists of three switchboards which are connected in a ring. Two generator sets are attached to each switchboard. The bus tie breakers are closed in this case study. The power consumed by the vessel is separated in two parts:

- DP loads: a load dependent on the thruster force.

- Non-DP loads: a constant load, which may include drilling and hotel loads.

The non-DP loads are prioritized above the DP loads in this case setup. The DP loads will therefore be reduced first and then the non-DP loads. This priority is relevant for operation where non-DP loads are most important, this is the case for cruise vessels and drilling vessels with active heave compensators. The method also allows other priorities, such as reducing non-DP loads before DP loads.

The safety requirement is that the surge position of the vessel should stay within $\pm 5 \mathrm{~m}$.

\section{MODEL}

The DP controller is modeled as a PID controller which is saturated by the power available signal, and backcalculation is used as an anti-wind-up method. The power available signal is used to avoid blackout by limiting the amount of power used by the consumers. The power consumed by the thrusters is assumed to be given by (Johansen et al., 2004):

$$
P_{D P}=K_{T 2 P}\left|T_{D P}\right|^{3 / 2}
$$

where $T_{D P}$ is the thruster force and $K_{T 2 P}$ is a constant depending on the thrusters.

The model for the vessel is made from the Marine Systems Simulator (Perez and Fossen, 2009). The model is reduced 
to only include motion in surge to simplify the example case. The equation for motion in surge is therefore:

$$
m \ddot{x}=-c\left(\dot{x}-u_{c}\right)+T_{D P}-T_{w i n d},
$$

where $m$ is the added mass and rigid body mass of the vessel in surge, $c$ is the linear damping coefficient, $u_{c}$ is the velocity of the ocean current, $T_{D P}$ is the thrust from $\mathrm{DP}$, and $T_{\text {wind }}$ is the wind force.

The governors of the generator sets are running in droop, with identical droop curves except when a new generator is connected and ramped in. This will make sure that the generator produces the same amount of power in per unit. The fuel consumption of each diesel engine is modeled using a Willans approximation (Guzzella and Onder, 2010):

$$
F C=a_{0}+a_{1} t_{m}+a_{2} t_{m}^{2},
$$

where $F C$ is the fuel consumption, $a_{i}$ are constants, and $t_{m}$ is the mechanical torque out of the diesel engine.

The typical fault which will be considered is a short circuit of one of the three switchboards. The chain of events after the short circuit is as follows:

(1) At the fault time the bus tie breakers connected to the failing switchboard are opened, while all other bus tie breakers are kept closed.

(2) After 200 milliseconds, the power available is reduced. The load is reduced such that each generator produces the same power as before the fault. In this study, the power will be reduced first by DP and secondly by non-DP loads.

(3) The power available is then increased as a ramp with slope of 5 percent per second, up to the maximum allowed overload $(110 \%)$.

(4) A new generator set is connected 45 seconds after the fault and with zero delivered power.

(5) The power available and the delivered power from the new generator are ramped up with a slope of 5 percent per second.

Remark 1. The power reduction is normally done by the thruster drives and other consumers and not by the DP, since the DP is too slow. However, the result of reducing the power available in this study versus demanding reduction of the load directly to the drives in a real vessel, gives the same response.

The fault is modeled by disconnecting Switchboard 1. In Table 1 the configurations of interest are listed. There are also many other possible configurations, but these are chosen such that they all have a unique number of generator sets connected after the fault and the minimum amount of generator sets connected before the fault.

Table 1. Number of generator sets connected to each switchboard (swbd) for each configuration.

\begin{tabular}{cccc}
\hline Configuration & Swbd 1 & Swbd 2 & Swbd 3 \\
\hline 1 & 1 & 1 & 0 \\
2 & 1 & 1 & 1 \\
3 & 2 & 2 & 1 \\
4 & 2 & 2 & 2 \\
\hline
\end{tabular}

In this study, only the total fuel consumption of the plant is used as cost function. It is calculated as the sum of the fuel consumption of each generator set, given by equation (3). However, it would be easy to include other cost functions, such as operational cost and startup/shutdown cost.

Parameters used in the simulations are given in Appendix A.

\section{SIMULATIONS}

\subsection{Case 1: Calm weather, high load}

In this case the ocean current is $0.1 \mathrm{~m} / \mathrm{s}$, the wind force is set to be equal the force of the current, and the power consumption from non-DP loads is set to $18 \mathrm{MW}$. The case is used to illustrate a situation where loss of power to non-DP loads is acceptable, and the weather is calm.

In Figure 2 and 3 results from the simulation of the case are shown. The simulations correspond to Configuration 1 to 3. With Configuration 1, non-DP loads are reduced with $33 \%$ until the stand-by generator set is connected and ramped up. The vessel will also drift-off $1.7 \mathrm{~m}$. This configuration can therefore only be selected if this is allowed; however, the fuel consumption is reduced with $2 \%$ compared with Configuration 2. Similarly, the fuel consumption is reduced with $8 \%$ and extra running hours of two generator sets are avoided if Configuration 2 is chosen instead of Configuration 3.

\subsection{Strong drift force, lower non-DP load}

In this case the current is set to $1.4 \mathrm{~m} / \mathrm{s}$, and non-DP loads to $1.4 \mathrm{MW}$. Results from this case are presented in Figure 4 and 5 .

It is sufficient to run with three generator sets, by choosing the optimal configuration the fuel consumption is reduced with $4 \%$.

\section{DISCUSSION}

The focus of this article has been to use simulation of faults to evaluate if a configuration is sufficient. This can be used as a decision support system, for the operator to select the best configuration. However, to be an alternative to load dependent start and stop tables, a method for taking the decision to start or stop a generator must be added to the implementation.

It is assumed that both power consumption from non-DP loads and environmental forces are constant. This is a good approximation as long as the weather is not too bad, since DP should only take care of drift forces. However, the power usage from drilling and other consumption can vary, for example can electrical heave compensators give large peaks for every wave crest. A method for establishing a worst-case simulation must also be made, for varying environmental forces and power consumption.

There are safety requirements other than position for some vessels. For example, it may be hard to synchronize additional generator sets to the switchboard if the frequency is varying due to high load variations. It could therefore be required that enough generator sets are connected to obtain small variations of the frequency. For other vessels, 

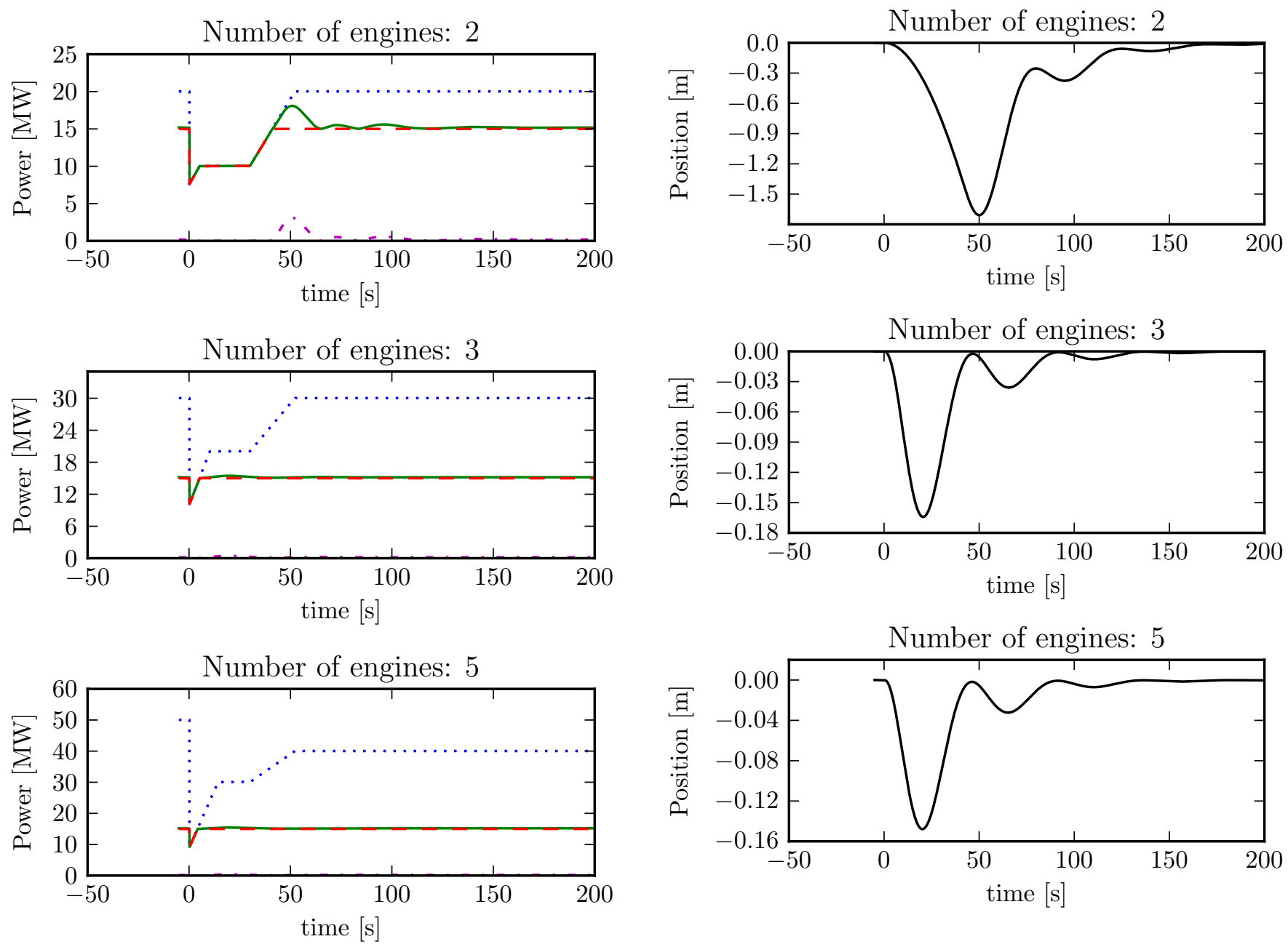

$$
\begin{array}{ll}
\hline \cdots & \text { Power Available } \\
- & \text { Power Total } \\
\text { - - } & \text { Power consumed by DP } \\
\text { - - } & \text { Power consumed by drilling } \\
\hline
\end{array}
$$

Fig. 2. Power consumption from simulation of fault for Case 1 with different configuration of the power plant. the position may be less important, while the angles of the risers are important. However, this method can include such safety requirements by including relevant subsystems in the model.

This method can be used with new upcoming technologies, such as DC-grid and battery packages. The model must however be adjusted to the actual system. Similarly, other types of blackout prevention can be used if that is desired.

One of the largest benefits of this approach is that the results from the simulations can be presented to the operator such that an informed decision can be made.

\section{CONCLUSION}

A decision support system for selecting a configuration of the power plant of a vessel is proposed. The system is based on simulations of worst-case failures; this is used to check which configurations are satisfying the safety

Fig. 3. Vessel position from simulation of fault for Case 1 with different configuration of the power plant.

requirements. A drilling ship in DP operation is used as a case plant to illustrate how the method works. The results from the simulations show that between 2 and $8 \%$ fuel can be saved, in addition to fewer running hours, by choosing the optimal configuration compared with a less optimal configuration.

\section{REFERENCES}

Contaxis, G.C. and Kabouris, J. (1991). Short term scheduling in a wind/diesel autonomous energy system. IEEE Transactions on Power Systems, 6(3), 1161-1167.

Det Norske Veritas (2011). Rules for Classification of Ships.

Dillon, T.S., Edwin, K.W., Kochs, H.D., and Taud, R.J. (1978). Integer programming approach to the problem of optimal unit commitment with probabilistic reserve determination. IEEE Transactions on Power Apparatus and Systems, PAS-97(6), 2154-2166.

Dokopoulos, P.S. and Saramourtsis, A.C. (1996). Prediction and Evaluation of the Performance of WindDiesel Energy Systems. IEEE Transactions on Energy Conversion, 11(2), 385-393.

Guzzella, L. and Onder, C.H. (2010). Introduction to Modeling and Control of Internal Combustion Engine 

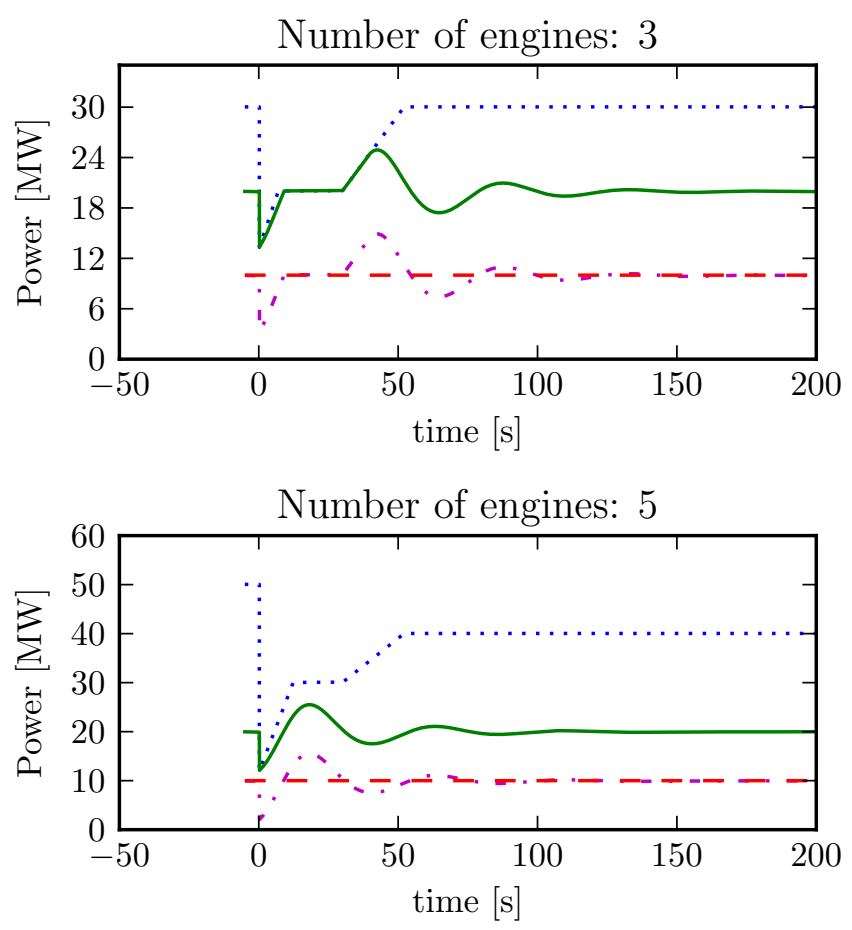

$$
\begin{array}{ll}
\cdots & \text { Power Available } \\
- & \text { Power Total } \\
-\cdot & \text { Power consumed by DP } \\
\text { - - } & \text { Power consumed by drilling }
\end{array}
$$

Fig. 4. Power consumption from simulation of fault for Case 2 with different configuration of the power plant.
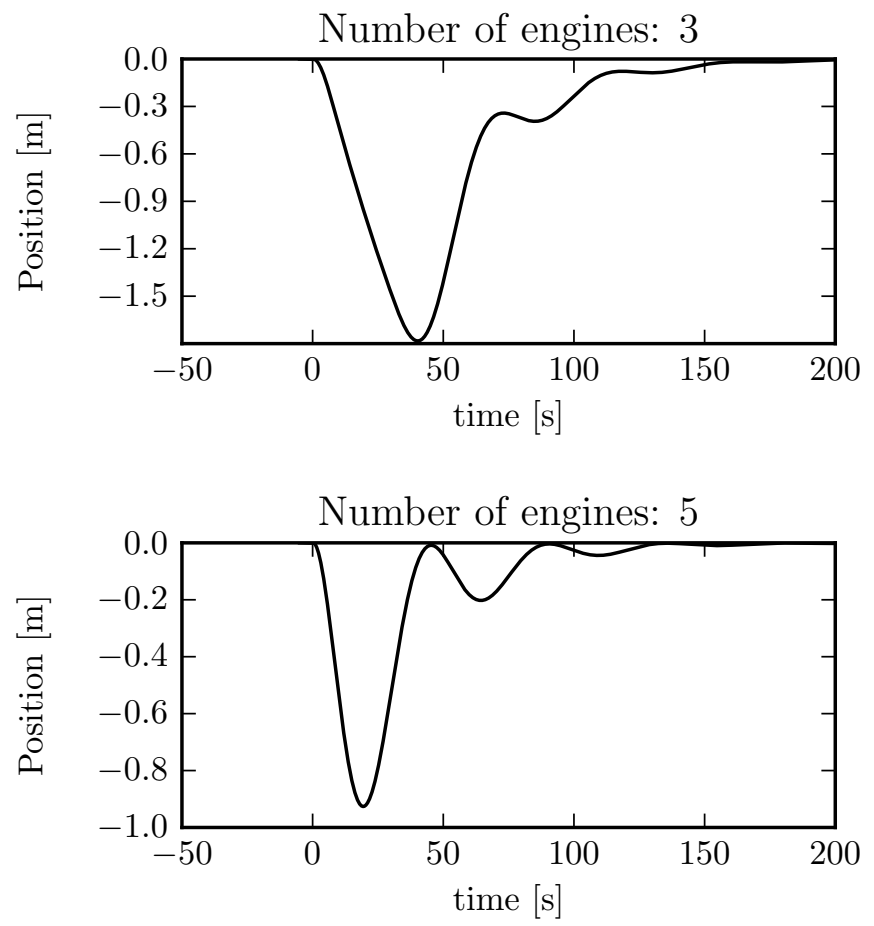

Fig. 5. Vessel position from simulation of fault for Case 2 with different configuration of the power plant.
Systems. Springer Berlin Heidelberg, Berlin, Heidelberg, second edition.

International Maritime Organization (1994). Guidelines for Vessels with Dynamic Positioning Systems. Maritime Safety Committee (MSC) Circular 645.

Johansen, T.A., Fossen, T.I., and Berge, S.P. (2004). Constrained Nonlinear Control Allocation With Singularity Avoidance Using Sequential Quadratic Programming. IEEE Transactions on Control Systems Technology, 12(1), 211-216.

Juste, K.A., Kita, H., Tanaka, E., and Hasegawa, J. (1999). An evolutionary programming solution to the unit commitment problem. IEEE Transactions on Power Systems, 14(4), 1452-1459.

Lowery, P.G. (1966). Generating unit commitment by dynamic programming. IEEE Transactions on Power Apparatus and Systems, PAS-85(5), 422-426.

Perez, T. and Fossen, T.I. (2009). A Matlab Toolbox for Parametric Identification of Radiation-Force Models of Ships and Offshore Structures. Modeling, Identification and Control: A Norwegian Research Bulletin, 30(1), $1-15$.

Radan, D. (2008). Integrated control of marine electrical power systems. Doctoral thesis, monograph, Norwegian University of Science and Technology.

Radan, D., Johansen, T.A., Sørensen, A.J., and Ådnanes, A.K. (2005). Optimization of Load Dependent Start Tables in Marine Power Management Systems with Blackout Prevention. WSEAS Transactions on Circuits and Systems, 4(12), 1861-1866.

Radan, D., Sørensen, A.J., Johansen, T.A., and Adnanes, A.K. (2006). Probability based generator commitment optimization in ship power system design. WSEAS Transactions on Systems, 5(8), 1901-1906.

Realfsen, B. (2009). Reducing NOx Emission in DP2 and DP3 Operations. In Dynamic Positionong Conference.

Senjyu, T. and Shimabukuro, K. (2003). A fast technique for unit commitment problem by extended priority list. IEEE Transactions on Power Systems, 18(2), 882-888.

Ådnanes, A.K. (2003). Maritime Electrical Installations and Diesel Electric Propulsion. Oslo, Norway.

Appendix A. SIMULATION PARAMETERS

\begin{tabular}{cc}
\hline Parameter & Value \\
\hline Mass + Added mass in surge & $43.74 \times 10^{6} \mathrm{~kg}$ \\
Linear damping & $437.4 \mathrm{kNs} / \mathrm{m}$ \\
$K_{T 2 P}$ & $0.0074 \mathrm{~W} / \mathrm{N}^{3 / 2}$ \\
$a_{0}$ & 0.0159 \\
$a_{1}$ & 0.1558 \\
$a_{2}$ & 0.0255 \\
\hline
\end{tabular}

